\title{
Lifetime Prevalence of Abortion and Risk Factors in Women: Evidence from a Cohort Study
}

\author{
Mehdi Moradinazar (D), Farid Najafi, Zeinab Moradi Nazar, Behrooz Hamzeh, Yahya Pasdar, \\ and Ebrahim Shakiba
}

Research Center for Environmental Determinants of Health (RCEDH), Health Institute, Kermanshah University of Medical Sciences, Kermanshah, Iran

Correspondence should be addressed to Ebrahim Shakiba; eshakiba@kums.ac.ir

Received 2 October 2019; Revised 2 February 2020; Accepted 21 February 2020; Published 27 April 2020

Academic Editor: Olav Lapaire

Copyright (c) 2020 Mehdi Moradinazar et al. This is an open access article distributed under the Creative Commons Attribution License, which permits unrestricted use, distribution, and reproduction in any medium, provided the original work is properly cited.

\begin{abstract}
Background. 10-20\% of pregnancies end due to spontaneous abortions. In recent years, nondocumentary evidence has been indicative of an increase in the prevalence of nonspontaneous abortions in Iran, especially in the Kurdish regions. The aim of this study is to assess the lifetime prevalence of spontaneous abortions and factors affecting spontaneous abortion in women 35-65 years old. Method. Data from the recruitment phase of Ravansar Non-Communicable Disease (RaNCD) cohort study was used. All of the 4831 married women 35-65 years old and with history of pregnancy were included in this study. In order to determine the abortion ratio, the number of abortions was divided by the number of live births, and multiple logistic regression analysis was applied to determine associated factors affecting abortion. Results. About $25.7 \%$ of women had a history of spontaneous abortion. The abortion ratio in women was 0.10 . The abortion ratio in women with secondary education, first pregnancy and marriage age at $\geq 26$, socioeconomic condition, and hyperthyroid and diabetes was high while the abortion ratio of women with high physical activity and BMI $<18.9$ or residents of rural area was low. After assessing the effective variables, it was found that women with high blood pressure have $63 \%$ less odds for nonspontaneous abortion, which is statistically significant $(p$ value $<0.05)$. Conclusion. Considering the effect of factors such as level of education, older age at the first marriage, and age at the first pregnancy on increased chance of spontaneous abortion, measures should be taken to take more care for these people.
\end{abstract}

\section{Introduction}

Spontaneous abortion is one of the most common complications of pregnancy [1]. In general, expulsion of an embryo or fetus before it reaches a stable stage of life is called abortion [2]. Studies indicate that the incidence of spontaneous abortion is between 10 and 20\% [3-5]. It should also be noted that most of the spontaneous abortions occur in the early weeks of pregnancy, and therefore, it can be confused with menstrual bleeding $[1,6,7]$. Generally, it is very difficult to determine the rate of spontaneous and unwanted abortions because in countries where legal abortion is prohibited, there is a possibility of false report. Besides, the study of spontaneous abortion in low- and middle-income countries is also very challenging because most abortions have not been reported to and recorded in their official health system [8].

In more than half of the cases, the causes of abortion have been genetic disorders and chromosomal abnormalities [9, 10]. Nevertheless, other factors affecting abortion are as follows: uterine abnormalities [11, 12], infectious diseases and untreated diseases of the mother $[1,4]$, the age of the mother during pregnancy, previous history of abortion $[1,13,14]$, age at the first menstruation [15], menstrual disorders [1], use of contraceptive drugs $[1,14], \mathrm{BMI}>25 \mathrm{~kg} / \mathrm{m}^{2}$ [16-19], environmental conditions and mother's lifestyle such as smoking [20,21] and use of caffeine [12, 13], being exposed to cigarette smoke $[22,23]$, stress $[12,24]$, exposure to mobile phone radiation [25], and low socioeconomic and 
employment status [26], which are effective in the occurrence of abortion.

Abortion is a distressing experience that affects the mother in a variety of ways by influencing on emotional status that can finally result in psychological disorders such as depression [27]. Although the prevalence of maternal abortion and miscarriage in Iran is stable, the number of new cases is slightly increasing. In fact, Iran is now facing with a decrease in population growth plus which is partly attributed to the increase in number of divorce and decrease in number of marriage among young men and women. While the nation is now trying to stabilize the growth of the population, a clear feature about the burden of abortion can help to understand the whole scenario. On the other hand, abortion is closely related to the cultural and religious factors, and Iran is a multicultural country with different ethnicities. Kurdish people mostly inhabited in western part of Iran with an integrated culture and life style. This study seeks to determine the lifetime prevalence of abortion and its risk factors in women 35-65 years old who have participated in the first cohort study among Kurdish people named Ravansar NonCommunicable Disease (RaNCD) cohort study.

\section{Methodology}

2.1. The Study Population. This cross-sectional study was conducted based on the population recruited for the RaNCD cohort study-a member of centers participated in Prospective Epidemiological Research Studies in Iran (PERSIAN). The recruitment phase began in November 2014 and ended in February 2017. During the course of this research, 10065 subjects willingly participated and signed the written informed consent letter. Further details have been presented elsewhere $[28,29]$.

2.2. Inclusion and Exclusion Criteria. Among all participating women, those with history of pregnancy were selected. In the RaNCD cohort study, the inclusion criteria for women were willingness to participate and complete the research, providing the signed written informed consent letter, and being capable of communication with the research team. For the purpose of this study, we excluded those who had no history of marriage and pregnancy.

2.3. Definition and Measurements. Socioeconomic status (SES), the main variable indicative of economic status of the family, was calculated by principal component analysis (PCA) and considered the subjects' wealth and social characteristics. Accordingly, the studied population was categorized into 5 quintiles: the poorest, the poor, the middle class, the rich, and the richest [30]. BIA device (InBody 770 BIOSPACE, Korea) was used for weight measurement. The heights measured with 0.1 accuracy using stadiometer [31]. A 19-item inventory related to light, moderate, and severe physical activities was used to collect information about the subjects' physical activity, and then, the Metabolic Equivalent of Task (MET) rate of each activity was obtained based on Compendium of Physical Activities to calculate daily MET rates of each participant. Physical activity levels were classi- fied as low (MET 24-36.5 hours per week), moderate (MET 36.6-44.9 hours per week), and heavy (MET $\geq 45$ hours per week) [32]. To measure the quality of nutrition, Healthy Eating Index (HEI) - based on the guidelines in 2015-was categorized into five groups [33]. In this study, any self-reported pregnancy ended spontaneously before week 20 is regarded as abortion. In order to determine the abortion ratio, the number of abortion was divided by the number of live births.

2.4. Statistical Methods. Continuous variables were mentioned as mean \pm standard deviation, and qualitative variables were measured by frequency (\%). In order to investigate about the risk factors of abortion, at first, a univariate logistic regression analysis was performed. Then, variables with $p<0.3$ were entered in the multiple model. Thereafter, variables with $p<0.05$ were kept, and other variables were excluded using a stepwise (Backward) method. In all of the analyses, missing values were deleted (less than 1\%). All the analyses were performed using the STATA V.14 (STATA Corp LLC) software. Pvalues $<0.05$ were considered as statistically significant.

\section{Results}

From of 4831 women participants, 2083 (58\%) of them were urban residents, and the rest were rural residents. 3472 $(72.2 \%)$ of them experienced their first menstrual bleeding when they were between 13 and 16 years old. The education level of 2202 (45.9\%) of the participants was between 1 and 5 years, and 3608 (74.8\%) women had no consanguineous marriage. In total, 1241 (25.7\%) had at least one spontaneous abortion during her life.

After adjustment for other variables, with the increase in the number of pregnancies, the risk for spontaneous abortion also increased; i.e., the odds for abortion in studied women who experienced more than 6 pregnancies was 8.3 (6.610.5) times significantly more than those with $1-3$ pregnancies. The odds of abortion in women who married after 26 years old was $1.6(1.02-2.4)$ times significantly more than the other. In addition, the risk of abortion in women who had their first pregnancy at age greater than 26 years old was $1.9(1.3-2.8)$ times significantly more than the others. Education level was an effective factor in spontaneous fetus abortion; i.e., with an increase in education level, the risk of abortion increased (Table 1). The highest abortion ratio was witnessed in women with secondary education (Figure 1).

From the total of women with past history of abortion, $24.8 \%$ had been using contraceptive pills. After adjusting the variables, it was shown that the use of pills is a protective factor for spontaneous abortion. Therefore, women who had been taking contraceptives pills had $22 \%$ less risk of abortion compared to those who had been using other contraceptive method which was statistically significant. Secondhand smoking nonsignificantly increased the odds ratio of abortion $=1.1$ (95\% C.I: 1.0-1.3). People who had the highest SES were in the greater risk of abortion (Table 1).

Compared to others, women with secondary education and those with marriage and first pregnancy age greater 
TABLe 1: Descriptive statistics of the sampled population and the results of the logistic regression model for risk factors of spontaneous abortion.

\begin{tabular}{|c|c|c|c|c|}
\hline Variables & $\begin{array}{c}\text { Total } \\
N(\%)\end{array}$ & $\begin{array}{c}\text { Abortion } \\
N \text { (prevalence) }\end{array}$ & $\begin{array}{c}\text { Abortion ratio } \\
\text { Mean (SD) }\end{array}$ & $\begin{array}{c}\text { Adjusted } \\
\text { OR }(95 \% \mathrm{CI})\end{array}$ \\
\hline Total (\%) & $4831(100)$ & $1241(25.7)$ & $0.10(0.2)$ & \\
\hline \multicolumn{5}{|c|}{ Menstruation start age } \\
\hline$<12$ years & $1031(21.4)$ & $276(26.8)$ & $0.11(0.2)$ & 1 \\
\hline $13-16$ years & $3472(72.2)$ & $891(25.7)$ & $0.11(0.2)$ & $0.9(0.8-1.1)$ \\
\hline$>17$ years & $306(6.4)$ & $64(20.9)$ & $0.07(0.1)$ & $0.7(0.5-1.0)$ \\
\hline \multicolumn{5}{|c|}{ Pregnancy number } \\
\hline $1-3$ & $1799(37.3)$ & $255(14.2)$ & $0.09(0.2)$ & 1 \\
\hline $4-5$ & $1376(28.5)$ & $420(30.5)$ & $0.14(0.3)$ & $4.5(3.7-5.5)$ \\
\hline$\geq 6$ & $1648(34.2)$ & $558(33.8)$ & $0.10(0.2)$ & $8.3(6.6-10.5)$ \\
\hline \multicolumn{5}{|c|}{ First pregnancy age (year) } \\
\hline 15 & $611(12.7)$ & $169(27.6)$ & $0.08(0.2)$ & 1 \\
\hline $15-20$ & $2469(51.2)$ & $620(25.1)$ & $0.09(0.2)$ & $0.9(0.8-1.2)$ \\
\hline $21-25$ & $1159(24.1)$ & $286(24.7)$ & $0.11(0.2)$ & $1.3(0.9-1.6)$ \\
\hline$\geq 26$ & $581(12.1)$ & $155(26.7)$ & $0.20(0.4)$ & $1.9(1.3-2.8)$ \\
\hline \multicolumn{5}{|c|}{ First marriage age (year) } \\
\hline$\geq 15$ & $1406(29.1)$ & $375(26.7)$ & $0.08(0.2)$ & 1 \\
\hline $16-20$ & $2338(48.4)$ & $593(25.4)$ & $0.10(0.2)$ & $1.2(0.9-1.4)$ \\
\hline $21-25$ & $728(15.1)$ & $174(23.9)$ & $0.12(0.2)$ & $1.2(0.9-1.6)$ \\
\hline$\geq 26$ & $358(7.4)$ & $98(27.4)$ & $0.19(0.4)$ & $1.6(1.0-2.4)$ \\
\hline \multicolumn{5}{|c|}{ Level of education } \\
\hline Illiterate & $1791(37.3)$ & $433(24.1)$ & $0.06(0.1)$ & 1 \\
\hline $1-5$ years & $2202(45.9)$ & $582(26.4)$ & $0.12(0.3)$ & $1.8(1.5-2.1)$ \\
\hline $6-9$ years & $461(9.6)$ & $123(26.8)$ & $0.14(0.3)$ & $2.4(1.8-3.2)$ \\
\hline $10-12$ years & $243(5.1)$ & $62(25.5)$ & $0.18(0.4)$ & $2.4(1.6-3.5)$ \\
\hline$\geq 13$ years & $111(2.1)$ & $41(36.9)$ & $0.23(0.3)$ & $3.5(2.1-5.8)$ \\
\hline \multicolumn{5}{|l|}{ Place } \\
\hline Urban & $2083(58.0)$ & $772(34.7)$ & $0.12(0.3)$ & 1 \\
\hline Rural & $1507(42.0)$ & $469(31.1)$ & $0.09(0.2)$ & $0.9(0.8-1.1)$ \\
\hline \multicolumn{5}{|c|}{ Consanguineous marriage } \\
\hline No & $3608(74.8)$ & $934(25.9)$ & $0.10(0.2)$ & 1 \\
\hline First degree & $678(14.1)$ & $172(25.4)$ & $0.11(0.3)$ & $0.9(0.8-1.2)$ \\
\hline Second degree & $541(11.1)$ & $135(2.9)$ & $0.09(0.2)$ & $0.9(0.8-1.2)$ \\
\hline \multicolumn{5}{|c|}{ Smoking status } \\
\hline No & $4548(94.4)$ & $1166(25.6)$ & $0.11(0.2)$ & 1 \\
\hline Current & $105(2.2)$ & $22(20.1)$ & $0.05(0.1)$ & $0.7(0.4-1.2)$ \\
\hline Former & $165(3.4)$ & $48(29.1)$ & $0.07(0.1)$ & $1.2(0.8-1.7)$ \\
\hline \multicolumn{5}{|c|}{ Secondhand smoking } \\
\hline No & $2446(50.6)$ & $599(24.5)$ & $0.10(0.2)$ & 1 \\
\hline Yes & $2385(49.4)$ & $642(26.9)$ & $0.11(0.3)$ & $1.1(1.0-1.3)$ \\
\hline \multicolumn{5}{|l|}{ BMI } \\
\hline$<18.9$ & $52(1.1)$ & $7(14)$ & $0.09(0.2)$ & 1 \\
\hline $19-24.9$ & $965(20.1)$ & $229(23.7)$ & $0.11(0.2)$ & $1.9(0.8-4.6)$ \\
\hline 25-29.9 & $1952(40.6)$ & $504(25.8)$ & $0.12(0.2)$ & $2.2(0.96-4.9)$ \\
\hline $30-34.9$ & $1381(28.8)$ & $380(27.5)$ & $0.09(0.2)$ & $2.4(1.1-5.5)$ \\
\hline$>35$ & $452(9.4)$ & $115(25.4)$ & $0.9(0.2)$ & $2.3(0.9-5.3)$ \\
\hline
\end{tabular}


TABLE 1: Continued.

\begin{tabular}{|c|c|c|c|c|}
\hline Variables & $\begin{array}{c}\text { Total } \\
N(\%)\end{array}$ & $\begin{array}{c}\text { Abortion } \\
N \text { (prevalence) }\end{array}$ & $\begin{array}{c}\text { Abortion ratio } \\
\text { Mean (SD) }\end{array}$ & $\begin{array}{c}\text { Adjusted } \\
\text { OR }(95 \% \mathrm{CI})\end{array}$ \\
\hline \multicolumn{5}{|l|}{ Physical activity daily METs } \\
\hline $24-36.5$ & $1036(21.4)$ & $261(25.2)$ & $0.11(0.3)$ & 1 \\
\hline $36.6-44.9$ & $3302(68.4)$ & $862(26.1)$ & $0.11(0.2)$ & $1.1(0.9-1.3)$ \\
\hline$\geq 45$ & $492(10.2)$ & $118(23.9)$ & $0.08(0.2)$ & $1.0(0.8-1.4)$ \\
\hline \multicolumn{5}{|l|}{ Use contraceptive drug } \\
\hline No & $812(16.8)$ & $240(29.5)$ & $0.13(0.3)$ & 1 \\
\hline Yes & $4013(83.2)$ & $996(24.8)$ & $0.10(0.2)$ & $0.8(0.6-0.9)$ \\
\hline \multicolumn{5}{|l|}{ Socioeconomic status } \\
\hline 1st quantile (the poorest) & $968(20.1)$ & $236(24.4)$ & $0.08(0.2)$ & 1 \\
\hline 2nd quantile & $966(20.0)$ & $222(22.9)$ & $0.09(0.2)$ & $0.9(0.7-1.1)$ \\
\hline 3rd quantile & $962(19.9)$ & $242(25.1)$ & $0.09(0.2)$ & $1.0(0.8-1.3)$ \\
\hline 4th quantile & $968(20.1)$ & $248(25.6)$ & $0.11(0.3)$ & $1.0(0.8-1.3)$ \\
\hline 5th quantile (the richest) & $962(19.9)$ & $291(30.2)$ & $0.15(0.3)$ & $1.4(1.1-1.8)$ \\
\hline \multicolumn{5}{|l|}{ Thyroid } \\
\hline No & $4546(94.1)$ & $1159(25.5)$ & $0.10(0.3)$ & 1 \\
\hline Нyро & $261(5.4)$ & $73(27.9)$ & $0.1(0.2)$ & $1.1(0.8-1.5)$ \\
\hline Hyper & $24(0.5)$ & $9(37.5)$ & $0.14(0.2)$ & $1.8(0.7-4.5)$ \\
\hline \multicolumn{5}{|l|}{ Blood pressure } \\
\hline No & $4465(92.7)$ & $1158(25.9)$ & $0.11(0.2)$ & 1 \\
\hline Yes & $353(7.33)$ & $76(21.5)$ & $0.12(0.3)$ & $0.6(0.5-0.8)$ \\
\hline \multicolumn{5}{|l|}{ Goodness of fit model } \\
\hline Sensitivity & $19.15 \%$ & & & \\
\hline Specificity & $95.25 \%$ & & & \\
\hline Accuracy & $75.97 \%$ & & & \\
\hline Positive predictive value & $57.79 \%$ & & & \\
\hline Negative predictive value & $77.63 \%$ & & & \\
\hline
\end{tabular}

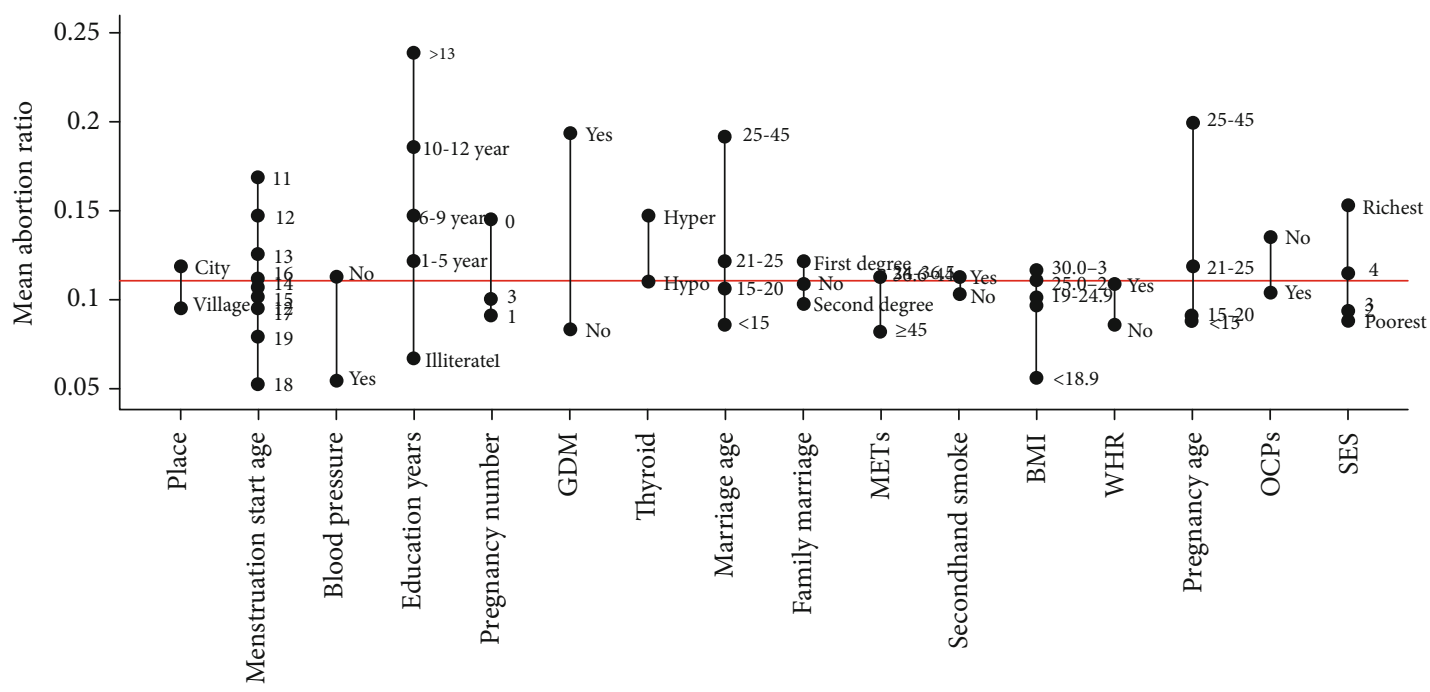

Risk factors of spontaneous abortion

FIGURE 1: Mean abortion ratio based on the studied variables in the participants of RaNCD. 
than 26 years, higher SES, past history of hyperthyroidism, and diabetes had a higher mean of abortion ratio. Also, in women with high blood pressure, heavy physical activity, and $\mathrm{BMI}<18.9$ as well as those who were living in rural areas, mean abortion ratio was less than the others (Figure 1).

\section{Discussion}

Although the total burden related to maternal abortion and miscarriage is less than $0.02 \%$ in Iran, the emotional complications of abortion (such as depression) as well as its physical complications may face the families and women with different psychosocial problems.

In the current research, the lifetime prevalence of spontaneous abortion was about $26 \%$ which is variable between 10 and $31 \%$ in different studies $[24,34,35]$. Despite the fact that the prevalence of abortion in this study was consistent with the previous researches, this result cannot be generalized to the whole society. In fact, abortion may occur in the early weeks of pregnancy when the mother is not aware of her pregnancy. In addition, in countries such as Iran in which the induced abortion is illegal, it is not easy to know about the exact burden of different types of abortion. In such situation, women hide the exact reason of abortion in order to take the advantage of hospital care.

The findings of this study suggested that age at the first marriage and age at the first pregnancy are important risk factors in spontaneous abortion. Accordingly, the risk for abortion in women with the first marriage and pregnancy at age greater than 26 years old was $57 \%$ and $87 \%$ more than the other age groups, respectively. This finding was also consistent with previous researches $[5,36]$. Scientists believe that marriage and pregnancy of a mother at older ages increase the risk of abortion, fetal and chromosomal problems, and pregnancy-related complications [37]. Therefore, as it has been recommended, it is necessary to have regular checkups and tests on the natural development of fetuses in pregnant women of older ages.

According to the findings of this research, secondhand smoking increased the risk for spontaneous abortion but not significantly. However, in similar studies, there was a significant relationship between increased abortion risk and secondhand smoking $[23,38]$. There is no single stage at which smoking is safe; thus, pregnant women should keep themselves away from exposure to tobacco contamination.

Contraceptive pills, as a preventive factor, decreased the abortion risk by $78 \%$ which was consistent with the findings of similar studies [39-41]. It may be because the contraceptive pills also have therapeutic effects, in addition to the contraceptive effect, and they are sometimes used to prevent ovarian cysts or to strengthen the follicles [42, 43]. There is also another therapeutic way to reduce abortion risk: the use of progesterone hormones prescribed by a gynecologist. Therefore, some hormonal contraceptive methods that contain progesterone may play a role in preventing spontaneous abortion [44].

In line with the findings of previous studies, the risk for abortion increased with the increasing number of pregnancies $[8,45,46]$.
The odds for spontaneous abortion increased with increasing level of education so the women with secondary education were at the greatest risk for spontaneous abortion. Many studies concluded that negative consequences of pregnancies were more evident in women with higher education $[47,48]$; however, a research in the northwest Ethiopia reached a conflicting result [36]. As women with secondary education decide to get married at older ages, factors such as older age at the first marriage as well as first pregnancy-a risk factor for spontaneous abortion-can increase the risk for abortion.

As shown in this study, the odds of abortion increased with the increasing SES. After adjusting the variables, it was found that pregnant women with higher SES are 1.36 times more likely to have abortion. In many of the previous studies, significant relationship was found between spontaneous abortion and SES [26, 49].

The prevalence of spontaneous abortion in women with hypertension was greater than in those without hypertension, and only $6.2 \%$ of the subjects who experienced spontaneous abortion had high blood pressure. After adjustment for other variables, it was found that women with high blood pressure were at a lower risk for spontaneous abortion. In the study conducted in Finland, no significant correlation was found between blood pressure and spontaneous abortion [35], and such finding might be due to the fact that hypertensive mothers get better health care which in turn helps to prevent abortion.

One of the limitations of this study was employing the self-report questionnaires to be completed by the subjects which may cause a problem in identifying spontaneous and nonspontaneous abortions. In Iranian culture and in area where people are still stick to the traditions such as where Kurdish people are living, families and women feel ashamed for any type of abortion. In addition, the induced abortion is illegal if there is no medical justification approved by forensic medicine and specialist. Such regulation contributes to not having an exact view regarding the true prevalence and the types of abortion. However, in the RaNCD cohort study, the investigator in line with the protocol of the PERSIAN cohort used a local and female interviewer for women, in order to get the correct answers to the questions. Such interviewer reassured the participants regarding the confidentiality of provided answers.

\section{Conclusion}

For countries such as Iran in which the psychosocial complication of abortion might be prominent, it is of great importance to know its risk factors within a population-based study. According to our results, as the number of pregnancies, age at the first marriage, age at the first pregnancy, and education level increase, the risk for spontaneous abortion also increases. While in line with socioeconomical development of women in Iran, all of such factors are increasing over the recent years, preventing abortion among such women in Iran is of great importance. In fact, strategies should be implemented through mass media, counseling, further education, and training about abortion and its risk factors to 
both people and medical care providers. While primary health care and maternal care given by both midwife and gynecologists have been provided for most of the cities all around Iran, a more high-quality care is needed for highrisk women.

\section{Abbreviations \\ PCA: Principal component analysis \\ SES: $\quad$ Socioeconomic status \\ RaNCD: Ravansar Non-Communicable Disease \\ HEI: Healthy Eating Index.}

\section{Data Availability}

All the information on how to access the RaNCD, with a list of current proposals and papers currently under preparation, can be found on our website: http://www.persiancohort.com.

\section{Ethical Approval}

The ethics committee of the Kermanshah University of Medical Sciences (KUMS.REC.1394.315) approved this study, and the written informed consent was obtained from each participant.

\section{Conflicts of Interest}

The authors declare that they have no conflicts of interest.

\section{Authors' Contributions}

M M, E SH, and FN participated in and conceptualized the study, collected the data, led the analysis, and wrote the text of the paper. MM and Y P advised on the conceptualization of the study, analysis of the data, and presentation of the results and reviewed and edited the text. EB and MM advised on the analysis of the data and presentation of the results and edited the text. All authors read and approved the final version of the manuscript.

\section{Acknowledgments}

This study was supported by the Ministry of Health and Medical Education of Iran and the Kermanshah University of Medical Science (Grant no: 92472).

\section{References}

[1] A. García-Enguídanos, M. E. Calle, J. Valero, S. Luna, and V. Domínguez-Rojas, "Risk factors in miscarriage: a review," European Journal of Obstetrics \& Gynecology and Reproductive Biology, vol. 102, no. 2, article S0301211501006133, pp. 111119, 2002.

[2] G. M. Stirrat, "Recurrent miscarriage I: definition and epidemiology," The Lancet, vol. 336, no. 8716, pp. 673-675, 1990.

[3] L. Sieiro Netto, C. Medina Coeli, E. Micmacher et al., "Influence of thyroid autoimmunity and maternal age on the risk of miscarriage," American Journal of Reproductive Immunology, vol. 52, no. 5, pp. 312-316, 2004.
[4] C. P. Griebel, J. Halvorsen, T. B. Golemon, and A. A. Day, "Management of spontaneous abortion," American Family Physician, vol. 72, no. 7, pp. 1243-1250, 2005.

[5] J. S. Cohain, R. E. Buxbaum, and D. Mankuta, "Spontaneous first trimester miscarriage rates per woman among parous women with 1 or more pregnancies of 24 weeks or more," BMC Pregnancy and Childbirth, vol. 17, no. 1, p. 437, 2017.

[6] J. F. Osborn, M. S. Cattaruzza, and A. Spinelli, "Risk of spontaneous abortion in Italy, 1978-1995, and the effect of maternal age, gravidity, marital status, and education," American Journal of Epidemiology, vol. 151, no. 1, pp. 98105, 2000.

[7] L. Regan and R. Rai, "Epidemiology and the medical causes of miscarriage," Best Practice \& Research Clinical Obstetrics \& Gynaecology, vol. 14, no. 5, article S1521693400901238, pp. 839-854, 2000.

[8] S. Dellicour, G. Aol, P. Ouma et al., "Weekly miscarriage rates in a community-based prospective cohort study in rural western Kenya," BMJ Open, vol. 6, no. 4, article e011088, 2016.

[9] D. Vaiman, "Genetic regulation of recurrent spontaneous abortion in humans," Biomedical Journal, vol. 38, no. 1, article 133777, pp. 11-24, 2015.

[10] K. Kleinhaus, M. Perrin, Y. Friedlander, O. Paltiel, D. Malaspina, and S. Harlap, "Paternal age and spontaneous abortion," Obstetrics \& Gynecology, vol. 108, no. 2, pp. 369377, 2006.

[11] P. C. Arck, M. Rücke, M. Rose et al., "Early risk factors for miscarriage: a prospective cohort study in pregnant women," Reproductive Biomedicine Online, vol. 17, no. 1, pp. 101-113, 2008.

[12] T. Li, M. Makris, M. Tomsu, E. Tuckerman, and S. Laird, "Recurrent miscarriage: aetiology, management and prognosis," Human Reproduction Update, vol. 8, no. 5, pp. 463-481, 2002.

[13] V. Dominguez-Rojas, J. R. de Juanes-Pardo, P. Astasio-Arbiza, P. Ortega-Molina, and E. Gordillo-Florencio, "Spontaneous abortion in a hospital population: are tobacco and coffee intake risk factors?," European Journal of Epidemiology, vol. 10, no. 6, pp. 665-668, 1994.

[14] E. de La Rochebrochard and P. Thonneau, "Paternal age and maternal age are risk factors for miscarriage; results of a multicentre European study," Human Reproduction, vol. 17, no. 6, pp. 1649-1656, 2002.

[15] K. Liestøl, "Menarcheal age and spontaneous abortion: a causal connection?," American Journal of Epidemiology, vol. 111, no. 6, pp. 753-758, 1980.

[16] C. Boots and M. D. Stephenson, "Does obesity increase the risk of miscarriage in spontaneous conception: a systematic review," in Seminars in Reproductive Medicine, pp. 507-513, Thieme Medical Publishers, 2011.

[17] M. Metwally, K. J. Ong, W. L. Ledger, and T. C. Li, “Does high body mass index increase the risk of miscarriage after spontaneous and assisted conception? A meta-analysis of the evidence," Fertility and Sterility, vol. 90, no. 3, pp. 714-726, 2008.

[18] M. Balsells, A. Garcia-Patterson, and R. Corcoy, "Systematic review and meta-analysis on the association of prepregnancy underweight and miscarriage," European Journal of Obstetrics \& Gynecology and Reproductive Biology, vol. 207, pp. 73-79, 2016. 
[19] Z. Veleva, A. Tiitinen, S. Vilska et al., "High and low BMI increase the risk of miscarriage after IVF/ICSI and FET," Human Reproduction, vol. 23, no. 4, pp. 878-884, 2008.

[20] A. Nielsen, C. Gerd Hannibal, B. Eriksen Lindekilde et al., "Maternal smoking predicts the risk of spontaneous abortion," Acta Obstetricia et Gynecologica Scandinavica, vol. 85, no. 9, pp. 1057-1065, 2006.

[21] F. Arffin, F. H. Al-Bayaty, and J. Hassan, "Environmental tobacco smoke and stress as risk factors for miscarriage and preterm births," Archives of Gynecology and Obstetrics, vol. 286, no. 5, pp. 1187-1191, 2012.

[22] J. D. Meeker, S. A. Missmer, D. W. Cramer, and R. Hauser, "Maternal exposure to second-hand tobacco smoke and pregnancy outcome among couples undergoing assisted reproduction," Human Reproduction, vol. 22, no. 2, pp. 337-345, 2007.

[23] J. D. Meeker, S. A. Missmer, A. F. Vitonis, D. W. Cramer, and R. Hauser, "Risk of spontaneous abortion in women with childhood exposure to parental cigarette smoke," American Journal of Epidemiology, vol. 166, no. 5, pp. 571-575, 2007.

[24] P. A. Nepomnaschy, K. B. Welch, D. S. McConnell, B. S. Low, B. I. Strassmann, and B. G. England, "Cortisol levels and very early pregnancy loss in humans," Proceedings of the National Academy of Sciences of the United States of America, vol. 103, no. 10, pp. 3938-3942, 2006.

[25] S. Kaur, J. Kaur, and M. Sandhu, "Effects of mobile radiations and its prevention," International Journal of Computer Science and Mobile Computing, vol. 5, p. 298, 2016.

[26] C. Arambepola, L. C. Rajapaksa, D. Attygalle, and L. Moonasinghe, "Relationship of family formation characteristics with unsafe abortion: is it confounded by women's socio-economic status?-a case-control study from Sri Lanka," Reproductive Health, vol. 13, no. 1, p. 75, 2016.

[27] H. Volgsten, C. Jansson, E. Darj, and A. Stavreus-Evers, "Women's experiences of miscarriage related to diagnosis, duration, and type of treatment," Acta Obstetricia et Gynecologica Scandinavica, vol. 97, no. 12, pp. 1491-1498, 2018.

[28] Y. Pasdar, F. Najafi, M. Moradinazar et al., "Cohort profile: Ravansar Non-Communicable Disease cohort study: the first cohort study in a Kurdish population," International Journal of Epidemiology, vol. 48, no. 3, pp. 682-683f, 2019.

[29] H. Poustchi, S. Eghtesad, F. Kamangar et al., "Prospective epidemiological research studies in Iran (the PERSIAN Cohort Study): rationale, objectives, and design," American Journal of Epidemiology, vol. 187, no. 4, pp. 647-655, 2018.

[30] H. Najafipour, M. Shokoohi, G. Yousefzadeh et al., "Prevalence of dyslipidemia and its association with other coronary artery disease risk factors among urban population in southeast of Iran: results of the Kerman coronary artery disease risk factors study (KERCADRS)," Journal of Diabetes \& Metabolic Disorders, vol. 15, no. 1, p. 49, 2016.

[31] Organization WH, "Obesity: preventing and managing the global epidemic: report of a WHO consultation on obesity, Geneva, 3-5 June 1997," Geneva: World Health Organization, 1998.

[32] W. Y. Craig, G. E. Palomaki, and J. E. Haddow, "Cigarette smoking and serum lipid and lipoprotein concentrations: an analysis of published data," BMJ, vol. 298, no. 6676, pp. 784788, 1989.
[33] S. M. Krebs-Smith, T. R. E. Pannucci, A. F. Subar et al., "Update of the healthy eating index: HEI-2015," Journal of the Academy of Nutrition and Dietetics, vol. 118, no. 9, pp. 1591-1602, 2018.

[34] J. X. Wang, M. J. Davies, and R. J. Norman, "Obesity increases the risk of spontaneous abortion during infertility treatment," Obesity Research, vol. 10, no. 6, pp. 551-554, 2002.

[35] E. Kharazmi, M. Fallah, and R. Luoto, "Miscarriage and risk of cardiovascular disease," Acta Obstetricia et Gynecologica Scandinavica, vol. 89, no. 2, pp. 284-288, 2010.

[36] A. S. Kebede, A. A. Muche, and A. G. Alene, "Factors associated with adverse pregnancy outcome in Debre Tabor town, Northwest Ethiopia: a case control study," BMC Research Notes, vol. 11, no. 1, p. 820, 2018.

[37] I. Nabti, R. Grimes, H. Sarna, P. Marangos, and J. Carroll, "Maternal age-dependent APC/C-mediated decrease in securin causes premature sister chromatid separation in meiosis II," Nature Communications, vol. 8, no. 1, article 15346, 2017.

[38] S. A. Venners, X. Wang, C. Chen et al., "Paternal smoking and pregnancy loss: a prospective study using a biomarker of pregnancy," American Journal of Epidemiology, vol. 159, no. 10, pp. 993-1001, 2004.

[39] Royal Collegeof General Practitioners' Oral Contraception study, "The outcome of pregnancy in former oral contraceptive users," BJOG: An International Journal of Obstetrics \& Gynaecology, vol. 83, no. 8, pp. 608-616, 1976.

[40] J. H. Ford and L. MacCormac, "Pregnancy and lifestyle study: the long-term use of the contraceptive pill and the risk of agerelated miscarriage," Human Reproduction, vol. 10, no. 6, pp. 1397-1402, 1995.

[41] S. Harlap, P. H. Shiono, and S. Ramcharan, "Spontaneous foetal losses in women using different contraceptives around the time of conception," International Journal of Epidemiology, vol. 9, no. 1, pp. 49-56, 1980.

[42] J. Russell, "Pre-cycle estrogen treatment and poor responders," Assisted Reproduction Review, vol. 5, pp. 82-89, 1995.

[43] P. Kovacs, P. E. Barg, and B. R. Witt, "Hypothalamic-pituitary suppression with oral contraceptive pills does not improve outcome in poor responder patients undergoing in vitro fertilization-embryo transfer cycles," Journal of Assisted Reproduction and Genetics, vol. 18, no. 7, pp. 391-394, 2001.

[44] N. Sakhavar, M. Mirteymouri, and B. Teymouri, "Effects of progesterone in threatened abortion," Journal of Babol University of Medical Sciences, vol. 10, no. 4, pp. 37-42, 2008.

[45] S. Cnattingius, L. B. Signorello, G. Annerén et al., "Caffeine intake and the risk of first-trimester spontaneous abortion," The New England Journal of Medicine, vol. 343, no. 25, pp. 1839-1845, 2000.

[46] X. Wang, C. Chen, L. Wang, D. Chen, W. Guang, and J. French, "Conception, early pregnancy loss, and time to clinical pregnancy: a population-based prospective study," Fertility and Sterility, vol. 79, no. 3, pp. 577-584, 2003.

[47] A. A. Sadiq, G. Poggensee, P. Nguku, K. Sabitu, A. Abubakar, and T. Puone, "Factors associated with adverse pregnancy outcomes and perceptions of risk factors among reproductive age women in Soba LGA, Kaduna State 2013," The Pan African Medical Journal, vol. 25, 2016. 
[48] G. Asiki, K. Baisley, R. Newton et al., "Adverse pregnancy outcomes in rural Uganda (1996-2013): trends and associated factors from serial cross sectional surveys," BMC Pregnancy and Childbirth, vol. 15, no. 1, p. 279, 2015.

[49] P. Rachootin and J. Olsen, "Prevalence and socioeconomic correlates of subfecundity and spontaneous abortion in Denmark," International Journal of Epidemiology, vol. 11, no. 3, pp. 245-249, 1982. 